\title{
Effects of heat transfer analysis through boson femtoscopy with fraction order differentiation
}

\author{
Ghulam Bary ${ }^{1 *}$, Waqar Ahmed ${ }^{2}$, Muhammad Sajid $^{3}$, Riaz Ahmad ${ }^{1}$, Ilyas Khan ${ }^{4 \dagger}$ \\ 1 Faculty of Science, Yibin University, Yibin 644000, China \\ 2 Department of Bionanotechnology, \\ Hanyang University, Ansan155-88, South Korea \\ 3 Faculty of Materials and Chemical Engineering, \\ Yibin University, Yibin 644000, China \\ 4 Department of Mathematics, College of Science Al-Zulfi, \\ Majmaah University, Al Majmaah 11952, Saudi Arabia
}

\begin{abstract}
Higher order femtoscopy measured to examine the heat exchanger characterization of the fluid debris produced in the collisions and investigated a remarkable suppression in the bosons interferences measurement. The analogous suppression can be analyzed to explore the coherence of boson thermal particle production sources at unprecedented energies. We illustrate the particles emissions from radiated sources with statistical coherence which induce the thermal particles interferences to probe the peculiarity of the heated sources as well as the distinctions about the heat exchangers in the collisions at higher temperature. We perspicacious that the bosons seem to the pertinent aspirant of heat exchanger, and the normalized three particles correlators evaluate the existence of such hybrid phases significantly. The key point of this research is that we analyze the three particles correlations with their normalized correlations by difference equations to determine the characteristics of heat exchanger and its applications. With such distinctive and efficient approach, we observe a significant difference in the correlation functions at higher temperature and momenta regimes.
\end{abstract}

Keywords: Femtoscopy, heat-mass transfer, fluid coherence fraction, radiated source characteristics

* ghulambary@gmail.com

$\dagger$ i.said@mu.edu.sa 


\section{INTRODUCTION}

The intensity interferometry is extensively used to explore the characteristics of the particle emitted fluids produced in the collisions of heavy nuclei at unprecedent energies [1-5]. The enormously used chaotic parameter lambda can be extracted by pertaining the assumption that the pions are ejected from the partially coherence heated sources in order to analyze the two pion femptoscopy. The considered parameter is lying between zero and one for the partially coherence particle production sources but possess unity for the fully thermal emissions $[1,2,6]$. Such parameter is correlated with the several other factors like the misidentification of particles, laser emission and the resonance decays of long lived $[1-5,7,8]$.

Perpetually, two boson interferences are evaluated by experimentally to explore the heat exchanger and coherence characterization, along with the peculiar information about the thermal energy particles emission sources. It has been observed that if the coherence fraction is quantified even more than fifty percent but the the chaotic thermal parameter suppression stipulated only twenty five percent. That is why it compelled the researchers that it is practically impossible to determine the coherence and chaotic properties of the particles emitted sources by using simple two particles statistical interferences alone [9]. Specifically, the higher order interferences possessed extra particulars which cannot be examine from the simple lower order correlations. The experimental measurements of the pions correlations at the famous world largest colliders performed and evaluated that the suppression of chaotic pion emissions which increases substantially at higher orders correlations than that of the lower order interferences [9-11].

In particular, the higher particle interferometry determines the source chaos-coherence fraction and also having several advance experimental dominance with elimination the influence of fourier transform phase and the decays of resonances which are long lived. [6, 12-16]. Three particle intensity interferences measured practically in the thermal collisions and the given results demonstrated that the normalized correlations of three particles revealed a crucial suppression from the chaos limit [15]. This obvious suppression of chaos limit may arise from the coherence fraction at low temperature and momenta regimes [16]. There are also various sources of non chaotic emissions such as the coherence of pions and the pulse radiation also provoke the coherence sources in the multiple form [17-19]. 
In addition, such investigations proclaim that the pions production sources created in the lead collisions at the extraordinary heat energies possess the partially coherence heat exchanger characteristics. Therefore the core point of this article is that we calculate the three particles correlations, genuine three particles interferences and the normalized correlation functions by consider the thermal particle emitted sources formed in the collision of heavy nuclei with completely as well as partially chaotic features and investigate the relationship between the particles number, temperature, heat energy, transverse momentum and the coherence fraction to explore the peculiar properties of the radiated sources less ambiguously than the usually measured simple two pion interferences. Such relationship examines the shape, size and the intrinsic properties of the particle ejecting sources which created during the impact of lead nuclei at the unusual energy. These correlations and the normalized correlations at various temperatures and transverse momenta cuts motivate to examine the fluid nature of the chaotic and coherence matter which created during the smashing of heavy nuclei at hadron colliders.

In this research, we explore the Bose Einstein coherence of indistinguishable bosons for particles ejecting gas source which expands relativistically inside the mean field potential of the harmonic oscillator ones that drops with time in hadronic phase temperature regime in the collisions of heavy nuclei. We compute the full three particles interferences, genuine correlations and the normalized interferences by using one and two particle density matrices within the hadronic phase temperature region about $60-170 \mathrm{MeV}$. The coherence influences of particle production source on the genuine correlations functions significantly in the interferometry measurements at various temperatures and momenta which are explored $[20,21]$.

The results reveal that the sources of thousands bosons could appear to own considerable degree of coherence which decrease the three particle statistical interferences and suppress the genuine three pions correlations and normalized three particles significantly at low transverse momenta but influence slightly on the genuine correlations at high temperaturemomenta regime. In the collisions of heavy nuclei at the hadron colliders, the copiously emission of particles can attain several thousands of pions and in this manifestation the substantial effects of coherence on the correlations and the corresponding normalized correlators in the multi particles interferences would be interesting.

This manuscript is organized into 5 sections. We investigate the source heat transfer 
fraction equations in Sec. II. In Sec. III, we calculate the correlations and normalized correlator functions. We analyzed our model results in Sec. IV. Finally, in Sec. V, we summarized our findings.

\section{SOURCE HEAT TRANSFER FRACTION EQUATIONS}

Bose Einstein condensation and thermalization are actually the macroscopic possession of the single quantum state of one particle and such condition habitually referred to the off diagonal prolonged range order. Bose Einstein condensation even though has been known long time due to its existence in various system like superfluid helium which verified in various laboratories [22]. We are curious in the analysis of boson gas of indistinguishable pions inside the potential of harmonic oscillator ones that commences from the hadronic medium mean field in the collision of heavy nuclei at higher energy [20, 21, 23]. The time dependent oscillator potential prescribed by

$$
V_{h o}(r, t)=\frac{1}{2} I_{e x}(t)\left(\frac{r}{l(t)}\right)^{2},
$$

where $I_{e x}(t)$ and $l(t)$ examine the strength $\hbar \omega(t)$ and characteristics length of considered harmonic oscillator, respectively. Here, we presume that the length $l$ is correlate to the expanding sources parameterized radius $R_{s}=\left(R_{i n}+v_{e x} t\right)$ which escalates with the evolution of time and $R_{\text {in }}$ represents the source initial radius, and the velocity parameter $v_{e x}$ related to expansion velocity of the considered source which will be examined by hydrodynamics relativistically. With the assumption that the time of source evolution is longer than the system relaxation time, we could approximately deal with such expanding gas like a unvaried system in every evolution time and the extension of the pion particles gas be possible to treat within the approximation under the process of adiabatic with quasi-static characteristics. For such expansion of gas the temperature $T$ of considered system and volume $V$ obey the intrinsic relation $T / V^{(1-\gamma)}$ equal to constant. The gamma quantify the ratio of specific heat capacity at constant pressures and constant volume.

For the ideal gas of boson particles, the energy of the system can be expressed as summing up of whole individual particles and the bosons energy levels for give potential strength $I_{e x}(t)=\hbar \omega(t)$ are

$$
\varepsilon_{n}=n I_{e x}+\frac{3}{2} I_{e x}, \quad n=0,1,2, \ldots
$$


The degeneracy of $D_{n}$ is $D_{n}=(n+1)(n+2) / 2$, and we are interested to built the mutual relations between the system size of hadronic gas and the temperature by relativistic hydrodynamics which deal with the collisions at higher order relativistic energies. The characterization of the system analyzed from hydrodynamic difference equations of spherical sources with initial radius $R_{i n}=6 \mathrm{fm}$ and the hadronized temperature $T_{h}=170 \mathrm{MeV}$. We explore that for the aforementioned considered source, the temperature of the system gratified that in the following way

$$
T=\frac{T_{h} R_{i n}^{\delta}}{\left(R_{i n}+v_{e x} t\right)^{\delta}}
$$

where $\delta=1.88$ and $v_{e x}=0.62$ [21]. We will utilize this temperature formula within our calculations which determined with relativistic hydrodynamics and the considered characteristic length $l$. We have been able to use the parameterized temperature formula in our computations of difference equations which developed through relativistic hydrodynamics for source investigations and will considered the characteristics length $l$ as

$$
l=K_{s}\left(R_{\text {in }}+v_{\text {ext }} t\right)
$$

where $K_{s}$ represents the proportional parameter which examine the root mean of the square radius. It is more crucial to magnify that in our calculations the parameter $K_{s}$ contained the values of 0.40 and 0.35 for the larger and small sources, respectively. The strength of the potential declines with time evolution which proceeds to decrease with the decreasing of temperature. At given temperature only small bosons numbers inhabit at the ground energy state and the others bosons distributed thermally in the higher energy states which proceed to cloud radius larger than the oscillator length. An approximation can be acquired by the considering $T>>I_{e x}$ and the system density deals with the Boltzmann distribution statistics. However, one can obtain temperature dependence coherence fraction at certain temperature $T<T_{c}$ and the particles obey the Bose-Einstein distributions [22].

In Fig. 1 we plot the root mean square radius (rmsr) of the considered source with the total number of pions $N_{T}=2000$ and 600 when the source size parameter contained $K_{s}=$ 0.35 and 0.40 . It is found that the rmsr results of the nuclear collisions at extraordinary energies are precise sensitive in the Lead-Lead collisions at LHC which having the rmsr of $10 \mathrm{fm}$, and in such measurements the the source sized parameters $K_{s}$ between the 0.35 and 0.40 represented reasonable the experimental results. 


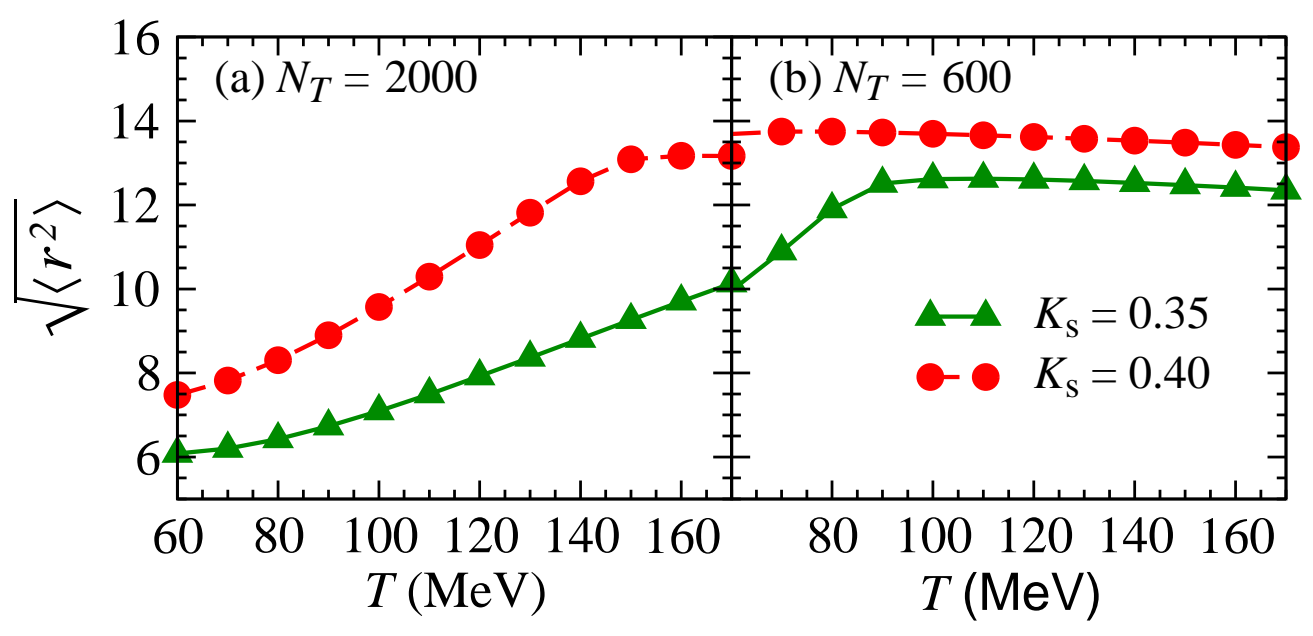

FIG. 1: Root mean square radius of the expanded sources versus temperature at different particles number $N_{T}$ and source size $K_{s}$.

\section{A. Effects of heat transfer with dependent coherence fraction}

The capability of Bose Einstein coherence to induce the pattern of interference with high visibility related closely with difference equations to examine the pion productions first order coherence. Recently, several experiments have been explored with trapped boson coherence gas and it is the aim of this article to investigate the coherence effect to the explanation of trapped pions gas. Our main focus will be the effect of coherence due to second and third order correlations with their corresponding normalized correlator. In the collisions of the nuclei at relativistic energy, the temperature of the hadrons gas phase is in the regimes of 60 to $170 \mathrm{MeV}$ and at these particular given temperatures the gesture of the motion of the considered bosons is treated relativistically because the particle mass is in the order to the considered temperatures. In such processes the particles system is treated as the static one at every small moment for the expansion of adiabatic quasi static, and the equation of the eigenvalue for the considered system can demonstrate as in Eq. (1) by following our previous discussion $[20]$

$$
\left[\frac{p^{2}}{2 m_{p}}+V_{h o}(r)\right] u(r)=\frac{E^{2}-m_{p}^{2}}{2 m_{p}} u(r)=\varepsilon u(r) .
$$

Here the potential $V_{h o}(r)$ of the oscillator is given by Eq. (1) at each time stage, for instance at the small duration. The eigenenergy of the relativistic pion is elucidated by

$$
E_{n}=\sqrt{m_{p}^{2}+2 m_{p}(n+3 / 2) I_{e x}}
$$


where $\mathrm{n}=0,1,2$.. and the ground state wave function for the lowest energy level can demonstrated as

$$
u_{c o}(r)=\left(\frac{1}{l^{2}(t) \pi}\right)^{3 / 4} e^{-\frac{r^{2}}{2 l^{2}(t)}} .
$$

For the considered crucial system of pions the $l(t)$ which illustrates time evolution function characteristics length and we present $E_{n}$ to measure the comparable energy levels from $n=0$ state to the excited energy gaps,

$$
\bar{E}_{n}=E_{n}-\sqrt{m_{p}^{2}+2 m_{p} \times \frac{3}{2} I_{e x}}
$$

For the identical boson gas of pions with a fixed number of particles, $N_{T}$, and at a given temperature $\mathrm{T}$ we can be write for such gas of indistinguishable boson particles at the specified temperature $T$ with fixed particles number $N_{T}$ in terms of coherence and chaotic particles

$$
N_{T}=N_{c o}+N_{c h},
$$

here, $N_{c o}$ represents the particles number in the coherence state where $m=0$,

$$
N_{c o}=\frac{Z}{1-Z} \text {. }
$$

Similarly, $N_{c h}$ illustrates the particles in the chaotic (excited) states where $m>0$,

$$
N_{c h}=\sum_{m>0}^{\infty} \frac{D_{m} Z e^{-\beta \tilde{E}_{m}}}{1-Z e^{-\beta \tilde{E}_{m}}},
$$

where $D_{m}$ expresses the degeneracy of the given $m$-th energy states and $Z$ denotes the parameter that contains the consequences of lower energy $\varepsilon_{0}$ but $\tilde{E}_{m}$ measure the comparative energy states from excited to ground (lowest) energy levels [20, 22]. The numerical values of fugacity $Z$ parameter lies between one and zero because the number of coherence particles $N_{c o} \geq 0$. With the help of aforementioned equations along with energy states of the given oscillator $[20,21]$, we can examine the parameter of fugacity $Z$ with fixed $N_{T}$ and afterwards can measure the coherence and chaotic fractions, respectively. Actually, Bose Einstein coherence arises when the system temperature is truncated below the particular temperature know as the critical temperature. In this matter, the number of coherence particles manipulated as $N_{c o} \sim N_{T}$ and $Z \sim N_{T} / N_{T}+1$.

In particular, we can write the coherence and chaos fractions in terms of the particles number and fugacity parameter,

$$
f_{c o}=\frac{N_{c o}}{N_{T}}=\frac{Z}{N_{T}(1-Z)}
$$




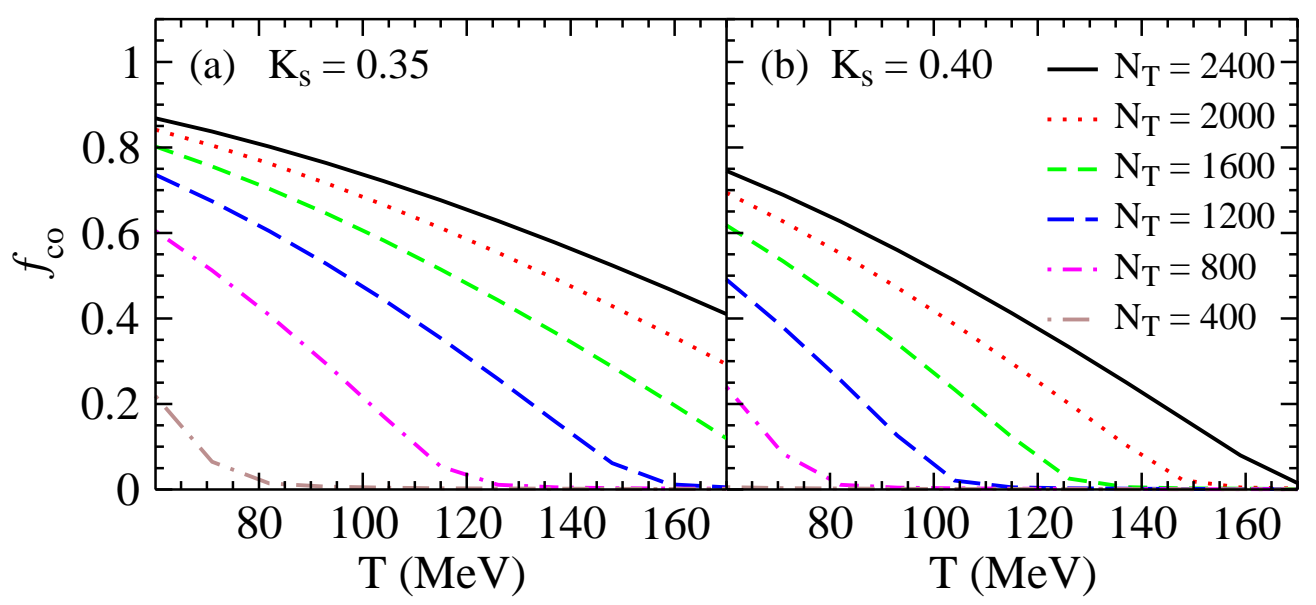

FIG. 2: Coherence fraction versus temperature $T$ for particle ejecting sources with distinct particles number $N_{T}$.

Fig. 2 presents the coherence fraction $f_{c}$ versus temperature $T$ for the sources contained the various total number of particles $N_{T}$ from 400 to 2400. The coherence fraction increased consistently with lowering the temperature and in the presence of large quantity of considered particles. However, the coherence fraction suppressed with increasing the temperature and reducing the particle quantity significantly. The plausible consequence is that the coherence fraction has peculiar relation with temperature $f_{c o}=1-\left[T / T_{h}\right]^{1.5}$. It is also meaningful to illustrate that the parameter $K_{s}$ play a vital role to describe the specification of the sources with particular parameterized values of 0.35 and 0.40 which illustrated in the left side and right side corresponding figures, respectively. The considered coherence is higher for small $K_{s}$ relative to large $K_{s}$ due to crucial consequences for the particles ejecting system possessed small scale of characterized length $l$ contained significant coherence effect at fixed $N_{T}$ and $T$. It is pretty obvious to indicate that the coherence fraction escalates with increasing $N_{T}$ but slumps from the specific tracks with rising the temperature.

$$
f_{c h}=\frac{N_{c h}}{N_{T}}=\frac{N_{T}-Z\left(N_{T}+1\right)}{N_{T}(1-Z)} .
$$

In the present research the coherence state refers to the system particular state with the properties of interferences which are absolutely close to possible to those specifically defined by classical field. In the most technical terms the successive orders of the coherence properties can be illustrated by the conditions of factorization which manipulate upon the succession of correlations functions field. 


\section{CORRELATION AND NORMALIZED CORRELATOR FUNCTIONS}

The preferred mechanism to investigate the properties of coherence are the higher order correlations. The coherence of first order depends on the specific properties of the single particle reduced density matrix which can be written in terms of the first order correlations functions and in the similar manner the coherence of second order relies by principle on the density correlations. It is more crucial to mention that the repulsion of short range among the various pions are not considered and the density correlations which are most supreme object of this study which plays a vital role for the the search of coherence properties. According to quantum statistics the one particle density matrix for pions for the given temperature and particle is

$$
G^{(1)}\left(k_{1}, k_{2}\right)=\sum_{m=0}^{\infty} u_{m}^{*}\left(k_{1}\right) u_{m}\left(k_{2}\right) \frac{D_{m} Z e^{-\beta \tilde{E}_{m}}}{1-Z e^{-\beta \tilde{E}_{m}}},
$$

where $u_{m}(k)$ represents the eigenfunctions of momentum space having exchange symmetry for oscillator potential and in the presence of large quantity of particles $N_{T}\left(N_{T}-1\right) \sim N_{T}^{2}(\gg$ $N_{c h}, N_{c o}$, the two particle density matrices can be illustrated as $[20,22]$

$$
\begin{aligned}
G^{(2)}\left(k_{1}, k_{2} ; k_{1}, k_{2}\right)= & G^{(1)}\left(k_{1}, k_{1}\right) G^{(1)}\left(k_{2}, k_{2}\right)+\left|G^{(1)}\left(k_{1}, k_{2}\right)\right|^{2} \\
& -N_{c o}^{2}\left|u_{c o}\left(k_{1}\right)\right|^{2}\left|u_{c o}\left(k_{2}\right)\right|^{2} .
\end{aligned}
$$

Therefore the two particle correlations can be represented as

$$
C\left(k_{1}, k_{2}\right)=\frac{G^{(2)}\left(k_{1}, k_{2} ; k_{1}, k_{2}\right)}{G^{(1)}\left(k_{1}, k_{1}\right) G^{(1)}\left(k_{2}, k_{2}\right)} .
$$

From Eq. (15), we have

$$
C\left(k_{1}, k_{2}\right)=1+\frac{\left|G^{(1)}\left(k_{1}, k_{2}\right)\right|^{2}-N_{c o}^{2}\left|u_{c o}\left(k_{1}\right)\right|^{2}\left|u_{c o}\left(k_{2}\right)\right|^{2}}{G^{1}\left(k_{1}, k_{1}\right) G^{(1)}\left(k_{2}, k_{2}\right)} .
$$

where $N_{c o}$ and $u_{c o}$ present the number of particles and the wave function of lowest state, respectively. This is the general expression for the coherence, partially coherence and the chaotic characteristics of the particle ejection sources. Specifically, in the case of perfect coherence source when all particles exist in the coherence state (ground state, $N_{c o} \rightarrow N_{T}$, then the both terms of numerator cancelled out and the correlations becomes unity due to the absence of quantum interferences effect.

$$
C\left(k_{1}, k_{2}\right)=1+\frac{\left|G^{(1)}\left(k_{1}, k_{2}\right)\right|^{2}}{G^{1}\left(k_{1}, k_{1}\right) G^{(1)}\left(k_{2}, k_{2}\right)}-\frac{N_{c o}^{2}\left|u_{c o}\left(k_{1}\right)\right|^{2}\left|u_{c o}\left(k_{2}\right)\right|^{2}}{G^{1}\left(k_{1}, k_{1}\right) G^{(1)}\left(k_{2}, k_{2}\right)} .
$$


The emission of the particles with identical momenta obeys the correlation function as given below

$$
C(k, k)=2-\left(\frac{N_{c o}\left|u_{c o}(k)\right|^{2}}{G^{(1)}(k, k)}\right)^{2} .
$$

The above function is adamant by the fraction of local coherence density to total density should be reduce to the unity at everywhere to narrate the completely coherence of second order. In general, near the trap center it can be close to unity only where the density of the condensate is large but the properties of the coherence in the thermal cloud which contains outside to condensate having the local density zero which is not different from the from the uncondensed gas. However, for the perfect chaotic source which having almost all particles in the higher energy state in order to having the particles number, $N_{c o}<<N_{T}$ and the second fraction can be eradicate, and then the above expression manipulated as

$$
C_{2}\left(k_{1}, k_{2}\right)=1+\frac{\left|G^{(1)}\left(k_{1}, k_{2}\right)\right|^{2}}{G^{(1)}\left(k_{1}, k_{1}\right) G^{(1)}\left(k_{2}, k_{2}\right)} .
$$

This is the expression for the fully chaotic source and the correlations possess the numerical value two at the intercept $\left(k_{1}=k_{2}\right)$ which is also known as chaotic limit for two particle correlations. However, it is worth mentioning here that for partially coherence sources the correlation fluctuates between two and unity which are the limits of the chaotic and coherence sources, respectively. The coherence fraction, particles numbers and two particle interferences are the basic ingredients of the correlations at higher order. Now we can describe the correlation of two particles interferences with their corresponding correlators for aforementioned case and the above equation manipulated as

$$
C_{2}\left(k_{1}, k_{2}\right)=1+\Lambda_{2}\left(k_{1}, k_{2}\right)
$$

In particular, the mentioned two particles correlators $\Lambda_{2}\left(k_{1}, k_{2}\right)$ represents the interferences for single particle pair. Mathematically, the general correlators for two pions can be expressed as

$$
\Lambda_{2}\left(k_{\mu}, k_{\nu}\right)=\frac{\left|G^{(1)}\left(k_{\mu}, k_{\nu}\right)\right|^{2}-N_{c o}^{2}\left|u_{c o}\left(k_{\mu}\right)\right|^{2}\left|u_{c o}\left(k_{\nu}\right)\right|^{2}}{G^{(1)}\left(k_{\mu}, k_{\mu}\right) G^{(1)}\left(k_{\mu}, k_{\nu}\right)},
$$

where $\mu, \nu=1,2$ for two particles correlations. In above equations the two particle correlator related to coherence wave function $u_{c o}(k)$, density matrix $G^{(1)}\left(k_{\mu}, k_{\nu}\right)$, and the coherence particle number $N_{c o}$ which illustrated as, for the fully chaotic source $N_{c o}<<N_{T}$ and all particles are in the excited states and the correlator touch its chaotic limit. On the contrary, 
in the situation when all particles are in the ground (coherence) state and then the considered source characterized as the coherence source, and the interferences consequences will be negligible. The contributions of correlatiors becomes zero and correlations become unity.

Furthermore, we know that the higher symmetrized correlations contain the most extra particulars than the simple two particle interferences which motivate us to investigate the consequences of the chaotic and coherence fractions about the particle emission sources along with the information about the several size scales. We examine the three particle intensity interferences versus relative momenta which provide the strong clue for the occurrence about the chaotic and coherence matter phase transition and its evolution as given below

$$
C_{3}\left(k_{1}, k_{2}, k_{3}\right)=1+\Lambda_{2}\left(k_{1}, k_{2}\right)+\Lambda_{2}\left(k_{1}, k_{3}\right)+\Lambda_{2}\left(k_{2}, k_{3}\right)+\Lambda_{3}\left(k_{1}, k_{2}, k_{3}\right),
$$

where the second, third and forth terms represent the two particle correlators due to the exchange of two particles but the last expression indicates the quantum interference in which all the three particles participate. The chaotic limit for the three particle correlation $C_{3}$ is six and any deviation from the given limit shows the occurrence of the coherence component.

In addition, the cumulant (genuine) three particle correlations can also be obtained by removing all the contributions of two particles interferences to examine pure triplet correlation. The above equation can be transformed into genuine correlation function which is illustrated as

$$
c_{3}\left(Q_{3}\right)=1+\Lambda_{3}\left(k_{1}, k_{2}, k_{3}\right)
$$

In particular, the mentioned three particles correlators $\Lambda_{3}\left(k_{\mu}, k_{\nu}, k_{\phi}\right)$ represents the interferences for triplet interferences. Mathematically, the correlator for three particle interferences with coherence, chaotic and partially coherence characteristics can be manipulated as [24-29]

$$
\begin{aligned}
\Lambda_{3}\left(k_{\mu}, k_{\nu}, k_{\phi}\right)= & 2\left[\frac{G^{(1)}\left(k_{\mu}, k_{\nu}\right) \rho^{(1)}\left(k_{\nu}, k_{\phi}\right) G^{(1)}\left(k_{\phi}, k_{\mu}\right)}{G^{1}\left(k_{\mu}, k_{\mu}\right) G^{(1)}\left(k_{\nu}, k_{\nu}\right) G^{(1)}\left(k_{\phi}, k_{\phi}\right)}\right. \\
& +2 \frac{N_{c o}^{3}\left|u_{c o}\left(k_{\mu}\right)\right|^{2}\left|u_{c o}\left(k_{\nu}\right)\right|^{2}\left|u_{c o}\left(k_{\phi}\right)\right|^{2}}{G^{1}\left(k_{\mu}, k_{\mu}\right) G^{(1)}\left(k_{\nu}, k_{\nu}\right) G^{(1)}\left(k_{\phi}, k_{\phi}\right)} \\
& -\frac{G^{(1)}\left(k_{\mu}, k_{\nu}\right) u_{c o}\left(k_{\mu}\right) u_{c o}\left(k_{\nu}\right)\left|N_{c o} u_{c o}\left(k_{\phi}\right)\right|^{2}}{G^{1}\left(k_{\mu}, k_{\mu}\right) G^{(1)}\left(k_{\nu}, k_{\nu}\right) G^{(1)}\left(k_{\phi}, k_{\phi}\right)} \\
& -\frac{G^{(1)}\left(k_{\nu}, k_{\phi}\right) u_{c o}\left(k_{\mu}\right) u_{c o}\left(k_{\phi}\right)\left|N_{c o} u_{c o}\left(k_{\mu}\right)\right|^{2}}{G^{1}\left(k_{\mu}, k_{\mu}\right) \rho^{(1)}\left(k_{\nu}, k_{\nu}\right) G^{(1)}\left(k_{\phi}, k_{\phi}\right)} \\
& \left.-\frac{G^{(1)}\left(k_{\mu}, k_{\phi}\right) u_{c o}\left(k_{\mu}\right) u_{c o}\left(k_{\phi}\right)\left|N_{c o} u_{c o}\left(k_{\nu}\right)\right|^{2}}{G^{1}\left(k_{\mu}, k_{\mu}\right) G^{(1)}\left(k_{\nu}, k_{\nu}\right) G^{(1)}\left(k_{\phi}, k_{\phi}\right)}\right],
\end{aligned}
$$


where $\mu, \nu, \phi=1,2,3$ for the three particles correlations functions. In above equations the two and three particles correlators related to coherence wave function $u_{c o}(k)$, density matrix $G^{(1)}\left(k_{\mu}, k_{\nu}\right)$, and the coherence particle number $N_{c o}$ which illustrated as, for the fully chaotic source $N_{c o}<<N$ and all particles are in the excited states and the correlations touch their chaotic limit. But in the situation when all particles are in the ground state and the considered source characterized as the coherence source, and the interferences consequences will be negligible. The contributions of correlatiors becomes zero. Hence the two and three particles interferences become one in the complete non chaotic source [21]. We can examine the density matrices with the evolving particle emission source at the step of every evolution for the given particles number $N_{T}$, and the temperature $T$ with the unique technique of $[20,21]$, and in this way we investigated the three particles intensity interferences for probe the nature of the particle ejected sources.

Moreover, the several other factors affect the momentum correlations which suggested that the mixed phase comprised of partially chaotic characteristics. It is more crucial to mention that if the pions are re dispersed after their ejection then the information will be lost in their evolution and to overcome such kind of re dispersing the investigation must be performed with long mean path in the considered matter. Therefore the evaluation about considered correlations would be intricate if the particles creation mechanism raised through the resonances of long lived. But these factors can be secluded by normalized three particles interferences to appropriate two particle interferences [16].

\section{A. Normalized three particle correlator}

The correlations of two particles are untrammelled about FT phase but it persists in the three particles correlations considerably. However, such kind of considered phase potentially isolate by weight up the cumulant interferences of three particles cumulant to two particle correlations. It is prevailed by the division of three particle correlator with under root of computed two particles correlators. Now we have been able to express the normalized three particle correlator $r_{3}$ for aforementioned source by using the expressions of two as well as three-pion correlators,

$$
r_{3}\left(Q_{3}\right)=\frac{c_{3}\left(Q_{3}\right)-1}{\sqrt{\Lambda_{2}\left(k_{1}, k_{2}\right) \Lambda_{2}\left(k_{1}, k_{3}\right) \Lambda_{2}\left(k_{2}, k_{3}\right)}} .
$$


Here, the mentioned correlators $\Lambda_{2}\left(k_{\mu}, k_{\nu}\right)$ and $\Lambda_{3}\left(k_{1}, k_{2}, k_{3}\right)$ represent the interferences for single particle pair and pure triplet, respectively [24-27].

We parameterized the chaotic strength and its stability in the form of normalized three particle correlator $r_{3}$ which touch to chaotic limit (two) for completely chaotic and undeviating system. The normalized correlator $r_{3}$ isolated the phase and the effect of resonance decay and it is precisely correlated with the chaotic and coherence fractions for the static sources $[25,30-33]$. In our model calculations the chaotic intercept $\Lambda_{2}\left(k_{1}, k_{2}\right)$ for the correlations of two particles when their corresponding relative momentum approaches to zero can represented as

$$
\Lambda_{2}(k)=1-\frac{N_{c o}^{2}\left|u_{c o}(k)\right|^{4}}{\rho^{(1)}(k, k)^{2}} \equiv 1-\left[f_{c o} f_{N}(k)\right]^{2},
$$

where $f_{c o}=N_{c o} / N_{T}$ and $f_{c h}=1-f_{c o}$ are the chaotic and coherence fractions,respectively. Particularly, the factor $f_{N}$ can magnified as [21]

$$
f_{N}(k)=N_{T}\left|u_{c o}(k)\right|^{2} / \rho^{(1)}(k, k)
$$

Now by substituting the values of Eqs. 22 and 25 into Eq. 26 at the vanishing relative momenta, and the normalized three particles correlator $r_{3}$ can be written as

$$
r_{3}(k, k, k)=2 \frac{1-3\left[f_{c o} f_{N}(k)\right]^{2}+2\left[f_{c o} f_{N}(k)\right]^{3}}{\left[1-\left[f_{c o} F_{N}(k)\right]^{2}\right]^{3 / 2}} .
$$

From above equations, it is quite clear that $f_{N}(k)$ gives the relative probability of particle pairs from the coherence state with momentum $k$ to the given momentum density. Therefore for the source which possessed the finite coherence fraction, $f_{N}(k)$ at the high momentum will decrease as the momentum $k$ increases because $\left|u_{c o}(k)\right|^{2}$ suppressed more quickly than $\rho(k)$ as $k$ rises and acquires its minimum value $f_{N}(k)=0$ at higher momentum. Therefore the behavior of the source at high momentum appears chaotic and $r_{3}=2$. The characteristics of the fluid ingredients details are comprised in [34-36]. These characteristics analyzed with the support of the Legendre polynomials with using the orthogonal properties and it has been observed that such polynomials directly related with the Hermite, Euler, Bernoulli and the Bernstein polynomials [37-39]. It is also perceive that the the coherence emissions influences on the correlations for the investigation of initial shape and size of the particles emitted fluids at the lower momentum [40,41]. On the other hand, the source behaves as a partially coherence at the lowest momentum and we can express the above expression in 
terms of chaotic fraction $f_{c o} F_{N}(k)=1-f_{c h}(k)$. In addition, the intercept of the normalized three particles correlator can be express in terms of the coherence fraction,

$$
r_{3}(k)=2 \sqrt{1-f_{c o}(k)} \frac{1+2 f_{c o}(k)}{\left(1+f_{c o}(k)\right)^{3 / 2}} .
$$

From this expression it is obvious that if the the source is completely chaotic i.e $f_{c o}=0$, then the the corresponding intercept of the $r_{3}$ attains its (chaotic limit) maximum value 2 . However, for a complete coherence source the coherence fraction $f_{c o}=1$ and the intercept of $r_{3}$ acquired minimum value (0) which is also know as coherent limit. $r_{3}$ fluctuates between zero and two for partially coherence particle ejected sources. Particularly, it is the pretty sensitive function of coherence fractions, particles momentum,temperature and particle numbers $N_{T}$.

\section{RESULTS AND DISCUSSION}

The main object of this research is to explore the nature of the peculiar radiated particle production sources which originated by the smashing of heavy nuclei at extraordinary energy and it can be feasible by using the three particles statistical correlations and their corresponding normalized correlator consistently in order to measure the nature of the heat exchanger. Here, we elucidate our model results about the partially coherence temperature dependent sources created in the collisions at higher energies and we presume that the produced sources consist on the debris of fluids which behave as temperature dependent sources. Fig. 3 presents the three particles correlations $C_{3}$ versus $Q_{3}$ for the considered expanding source with distinct temperatures at different size of the particle production sources with $K_{s}=0.35,0.40$ which contained $N_{T}=600-1200$. The chaotic limit of the $C_{3}$ is six at the intercept. The correlations increase with the reduction of number of particles $N_{T}$ and increasing the temperature $T$ for the small source size $K_{s}=0.35$ as shown left panels $(a-c)$. It is obvious that the correlations for the large source size $K_{s}=0.40$ influence small due the increasing the numbers of particles and decreasing the temperature because the large source size The results illustrate the peculiar properties at various temperatures from 80 to 120. The source behaves like a fully chaotic at higher temperatures and the correlations possess almost maximum values when the temperatures are in the range of $120 \mathrm{MeV}$ at small number of particles. The correlations $C_{3}$ decrease gradually with lowering the tem- 

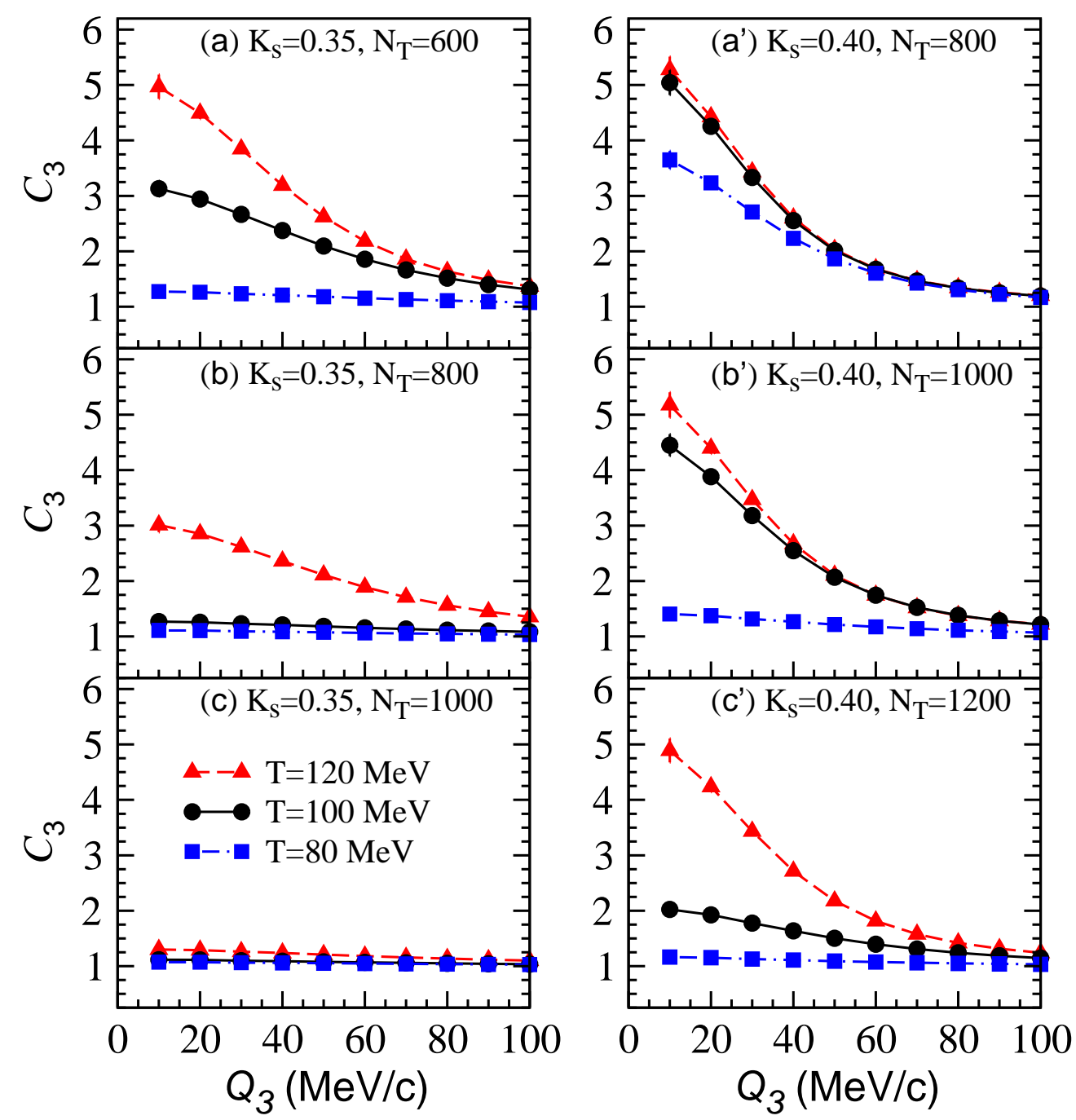

FIG. 3: Three particles correlations $C_{3}$ versus $Q_{3}$ with various temperatures and different particles number $N_{T}$.

perature because there exist coherence components at lower temperatures which effect the correlations significantly. The causes are pretty obvious that the sources contain lower coherence fractions at higher temperatures than that of the lower order temperatures because the source at higher temperatures creates energetic particles from the excited states which boost the correlations incoherently. The result of $C_{3}$ for the source with temperature 120 $\mathrm{MeV}$ approach to 1.4 due to the domination of coherence at lower temperatures whereas at higher temperatures the correlations touch to maximum chaotic limit. The correlations increase gradually with rising the temperatures and decreasing the numbers of particles. In addition, the particles ejected from the excited states possess the average momentum higher 


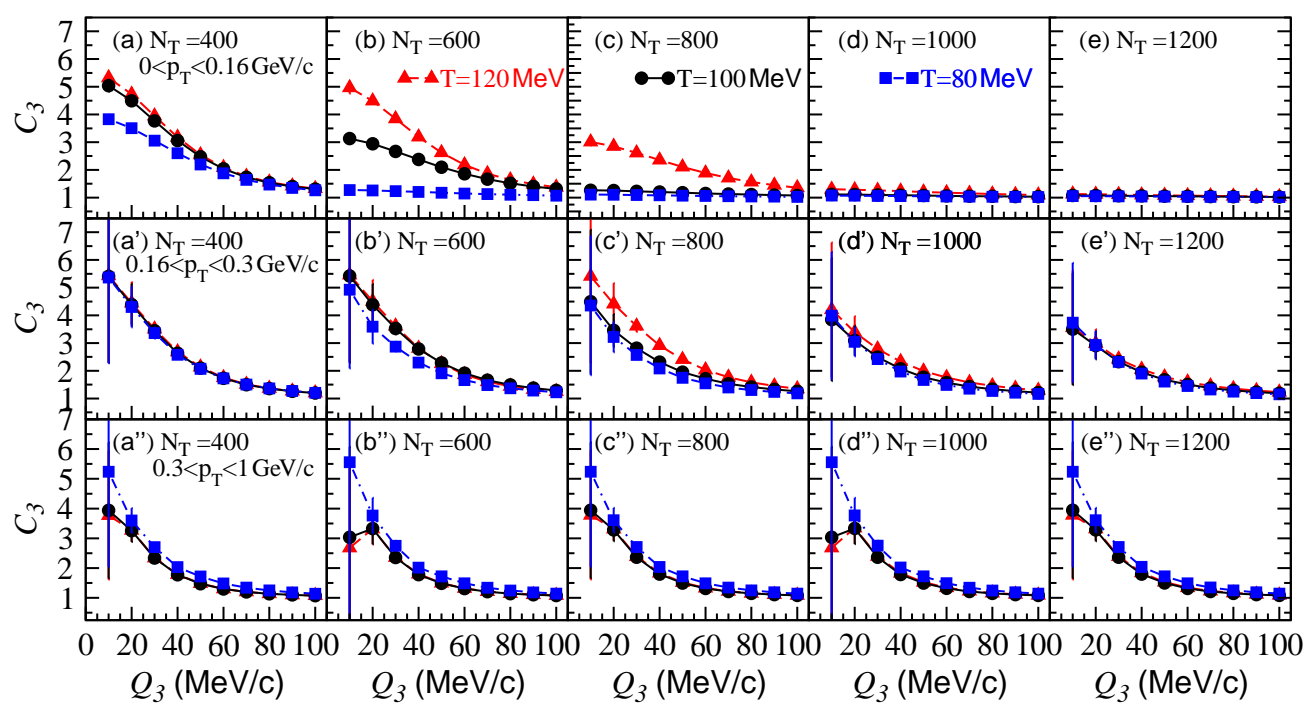

FIG. 4: Three particles correlations $C_{3}$ versus $Q_{3}$ for the small sources $K_{s}=0.35$ at three cuts of transverse momenta and number of particles $N_{T}$.

than those which emitted from the lower energy state (coherence emission) in the model of pion gas. Therefore the correlations functions for such sources with the possible finite coherence fraction are the momentum dependent.

We plot Fig. 4 for the three particles correlations functions versus $Q_{3}$ of the particle emission sources with $K s=0.35$ possessed the number of particles $N_{T}=400,600,800$, 1000 and 1200. The three cuts of transverse momenta have been applied during the calculations of our model results which are $0<p_{T}<0.16 \mathrm{GeV} / c, 0.16<p_{T}<0.3 \mathrm{GeV} / c$, and $p_{T}>0.3 \mathrm{GeV} / c$, respectively. The results are shown in Figs. 4(a)-(e), Figs. 4( $\left(a^{\prime}\right)-\left(e^{\prime}\right)$, and Figs. 4(a")-(e"), respectively. Figs. 4(a)-(e) illustrate the correlations at lower transverse momentum $0<p_{T}<0.16 \mathrm{GeV} / c$ and the results express the significant decline at lower temperature and higher multiplicity. The results increase with the temperature $T$ of the particle ejected source and decrease gradually by increasing the particle numbers $N_{T}$. The reasons are quite obvious that the emission sources at higher temperature possess the lower condensation fraction. The effect of condensation rises abruptly with decreasing the temperature and increasing the number of particles. The correlations for the considered source with higher temperature and small number of particles at the intercept touch the chaotic limit six but decrease with increasing the number of particles ( 400 to 1200) and lowering temperature from $120 \mathrm{MeV}$ to $80 \mathrm{MeV}$. Such behavior represents the indication of chaotic source at higher temperature and the results at $T=80 \mathrm{MeV}$ indicate the considerable con- 


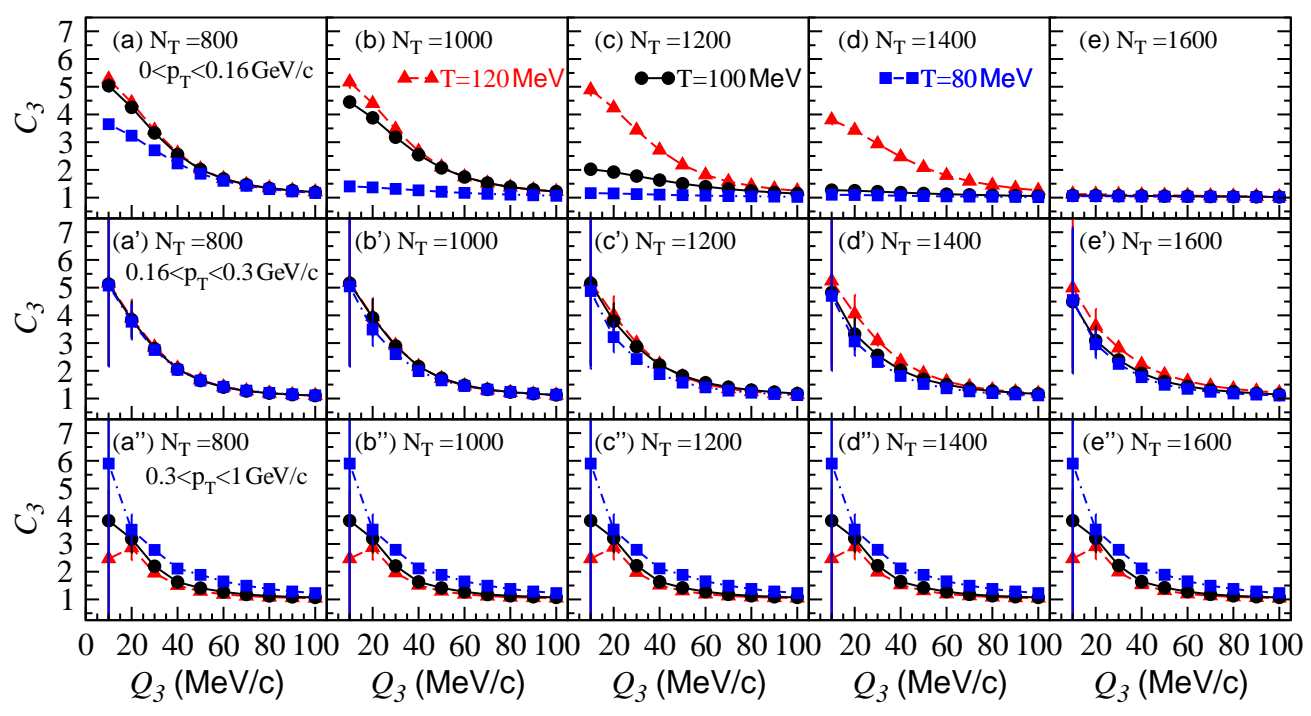

FIG. 5: Three particles correlations $C_{3}$ versus $Q_{3}$ for the sources $K_{s}=0.40$ at three cuts of transverse momenta with different particles number $N_{T}$.

densation of the coherent emission. However, Fig. 4(e) specify that the considered sources with the three different temperatures possess high coherent fractions when $N_{T}=1200$.

On the other hand, at the highest transverse momenta interval $p_{T}>0.3 \mathrm{GeV} / c$ as illustrated in [Figs. 4( $\left.a^{\prime \prime}\right)-\left(e^{\prime \prime}\right)$ ], the correlations at the intercept shows that almost all particles ejected chaotically with the higher momenta even the considered sources which having the high coherence fraction due to high particles multiplicity $N_{T}$ and lower temperature $T$. It is notable that the widths of the correlations functions at low transverse momenta represent wider behavior than those of the correlations functions measured at highest transverse momenta because the pions ejected sources have wide spatial distributions for the pions ejected from the excited state.

The correlations for considered sources at higher temperature are slightly lower than those of $T=80 \mathrm{MeV}$ due to fact that the spatial distributions are narrower at the lower temperatures for the chaos emission ejected from the excited states. In particular, the effect of the coherence fraction on the correlations is considerably little as shown in the middle momentum interval $0.16<p_{T}<0.3 \mathrm{GeV} / c$ then those at lower transverse momenta because the ejection of pions from excited states are numerically larger that those at the lowest momentum. The impact of spatial distributions also play a vital role on the correlations at various temperatures as shown in the momentum dependent middle panels.

Fig. 5 illustrate the correlations of three particles $C_{3}$ versus relative momenta $Q_{3}$ which 
is invariant for the evolving sources contained pion gas with the parameter $K_{s}=0.40$ and particles numbers $N_{T}=800,1200,1400,1600$. The cuts of the transverse momenta are imposed as $p_{T}<0.16 \mathrm{GeV} / c, 0.16<p_{T}<0.3 \mathrm{GeV} / c$ and $p_{T}>0.3 \mathrm{GeV} / c$ respectively. The top horizontal panels shows the variations of correlations with different particles which increased from left to right gradually. It is obvious that at the lower transverse momenta the correlations decrease with increase the numbers of particles at fixed temperature as shown in the Figs. 5(a)-(e). The correlations increase with rising the temperature by fix the particles numbers and decrease with lowering the temperature, but the effect of coherence appears significantly at lower $T=80 \mathrm{MeV}$ when the number of particles are 1600 due to the Bose Einstein condensation. One can observe that the behavior of the correlations is almost same as those of the small sources when $K_{s}=0.35$. However, the correlations at the higher transverse momenta appear the temperature and number of particles independent because the the particles with highest momentum ejected chaotically from the higher energy states which compelled the bosons to correlate chaotically and touch to the chaotically as shown the last and second last horizontal panels of Fig. 5.

Fig. 6 presents the cumulant (genuine) three particles correlation $c_{3}$ versus $Q_{3}$ for the considered expanding sources with distinct temperatures $\mathrm{T}$ and particle numbers $N_{T}$. The three pions genuine correlations for the given sources contained the numbers of particles $\left(N_{T}=600,800\right.$ and 1000 for the sources of small size $K_{s}=0.35$ as illustrate in the Figs. 6(a)-(c). The correlations of the three particles decrease by increasing the numbers of particles when we fixed the temperature because large numbers of particles have maximum probability to reside coherently at the lower temperatures. The lowering the temperature at fixed number of particles cause to decrease the thermal energy of the particles continuously $K . E \propto T$ and bosons condense at the lower energy state, and form the Bose-Einstein condensations which effect the bosons correlations significantly.

It is more worthy to mention that for the small sources $\left(K_{s}=0.35\right)$ the effect of the intensity interference appear to zero when the number of particles are 1000 as shown in the Fig. 6(c). Figs. 6(a')-(c') illustrate the genuine correlations of three considered bosons when the given source has pretty large size $(K s=0.40)$ which possess the particles numbers $N_{T}=800,1000$, and 1200. The correlations decrease by increasing $N_{T}$ because the considered source with large number of particles possesses the significant coherence effect. The correlations increase with rising the temperature for fix the number of particles $N_{T}$ because at higher temperatures 

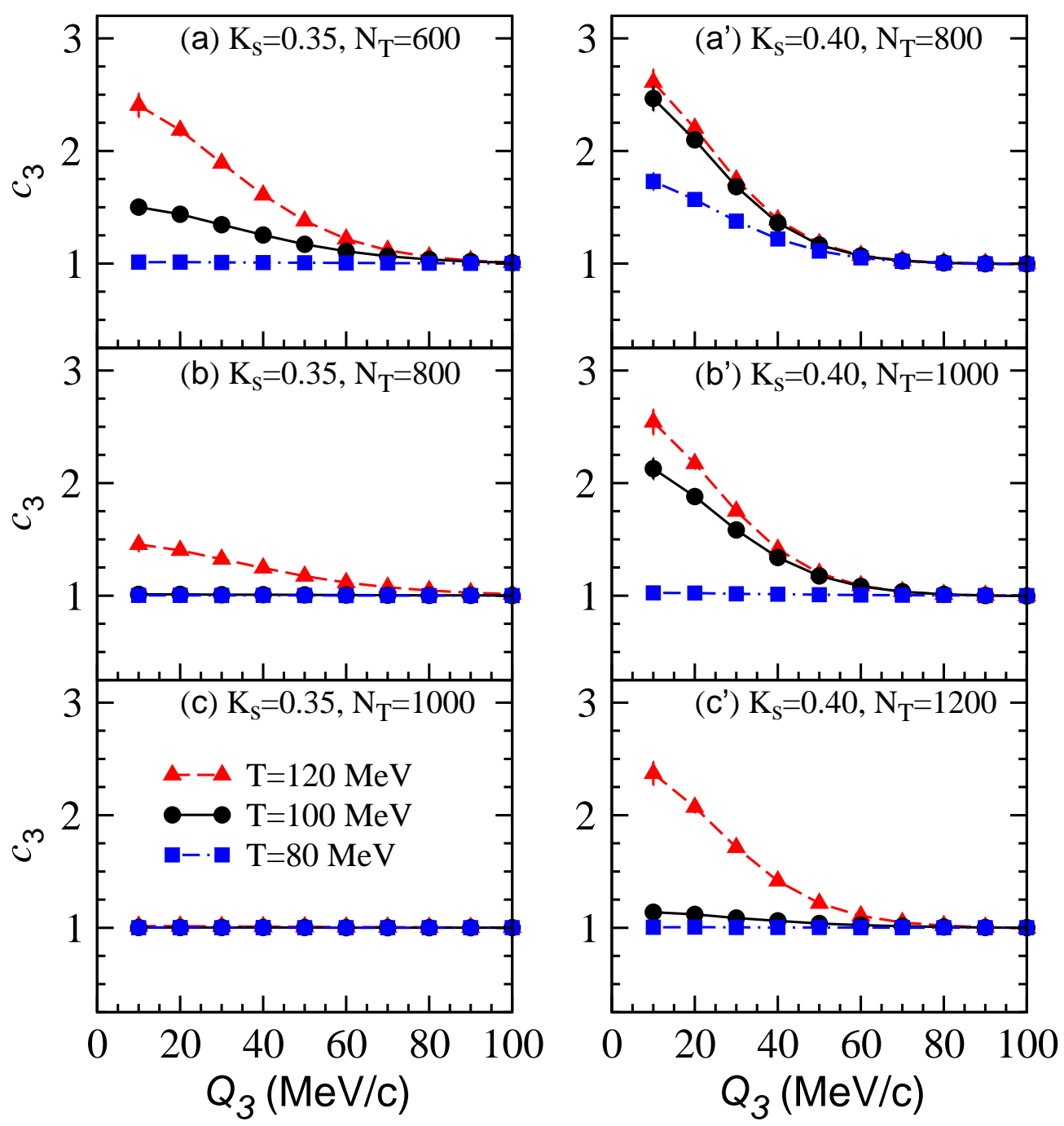

FIG. 6: Three particles cumulant correlations $c_{3}$ versus $Q_{3}$ at various transverse momenta with different particles number $N_{T}$.

the coherence is lower significantly and correlations approach to the chaotic limit three at the intercepts. In particular, the genuine three particles correlations are pretty lower at higher temperatures for the fixed number of particles $N_{T}$ for both sources. It is quite clear that the correlations of three particles for the considered sources with $K_{s}=0.40$ have an enhancement than for the sources with the parameter $K_{s}=0.35$ by fix the number of particles $N_{t}$ and temperature $T$. This cause happen due to fact that the source with large parameter $K_{s}$ has the large characteristics lengths and lower coherence fraction.

Fig. 7 shows the genuine three particles correlations functions versus $Q_{3}$ of the particle emission sources with $K s=0.35$ having the number of particles $N_{T}=400,600,800$, 


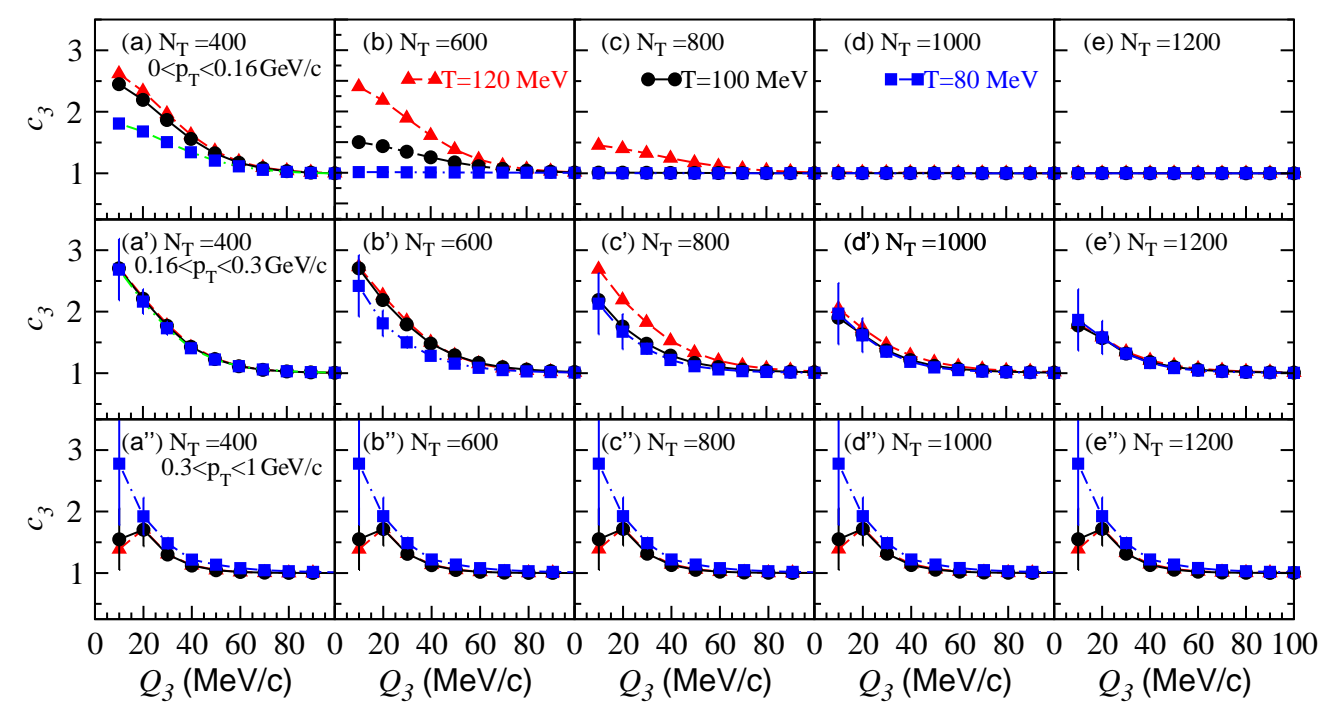

FIG. 7: Three particles cumulant correlations $c_{3}$ versos $Q_{3}$ for the small sources $K_{s}=0.35$ at various transverse momenta with different particles number $N_{T}$.

1000 and 1200. The three cuts of transverse momenta have been applied during the calculations of our model results which are $0<p_{T}<0.16 \mathrm{GeV} / c, 0.16<p_{T}<0.3 \mathrm{GeV} / c$, and $p_{T}>0.3 \mathrm{GeV} / c$, respectively. The results are shown in Figs. 7(a)-(e), Figs. 7( $\left.a^{\prime}\right)-\left(e^{\prime}\right)$, and Figs. 7( $\left.a^{\prime \prime}\right)-\left(e^{\prime \prime}\right)$, respectively. Figs. 7(a)-(e) denote the correlations at lower transverse momentum $0<p_{T}<0.16 \mathrm{GeV} / c$ and the results express the significant decline at lower temperature and higher multiplicity. The results of genuine correlations increase with the rising temperature $T$ and touche to the chaotic limit three of the particle ejected source and decrease gradually by increasing the particle numbers $N_{T}$. The reasons are quite obvious that the emission sources at higher temperature possess the lower condensation fraction.

The effect of condensation boost up abruptly with decreasing the temperature and increasing the number of particles. The correlations for the considered source with higher temperature and small number of particles at the intercept touch the chaotic limit three but decrease with increasing the number of particles ( 400 to 1200), and lowering temperature from $120 \mathrm{MeV}$ to $80 \mathrm{MeV}$. Such behavior represents the indication of chaotic source at higher temperature. In particular, the results at $T=80 \mathrm{MeV}$ indicate the considerable condensation of the coherent emission for the considered small source. However, Fig. 4(e) describe that the considered sources with the three different temperatures possess high coherent fractions when the numbers of particles are pretty high $N_{T}=1200$.

In addition, at the highest transverse momenta interval $p_{T}>0.3 \mathrm{GeV} / c$ as illustrated in 


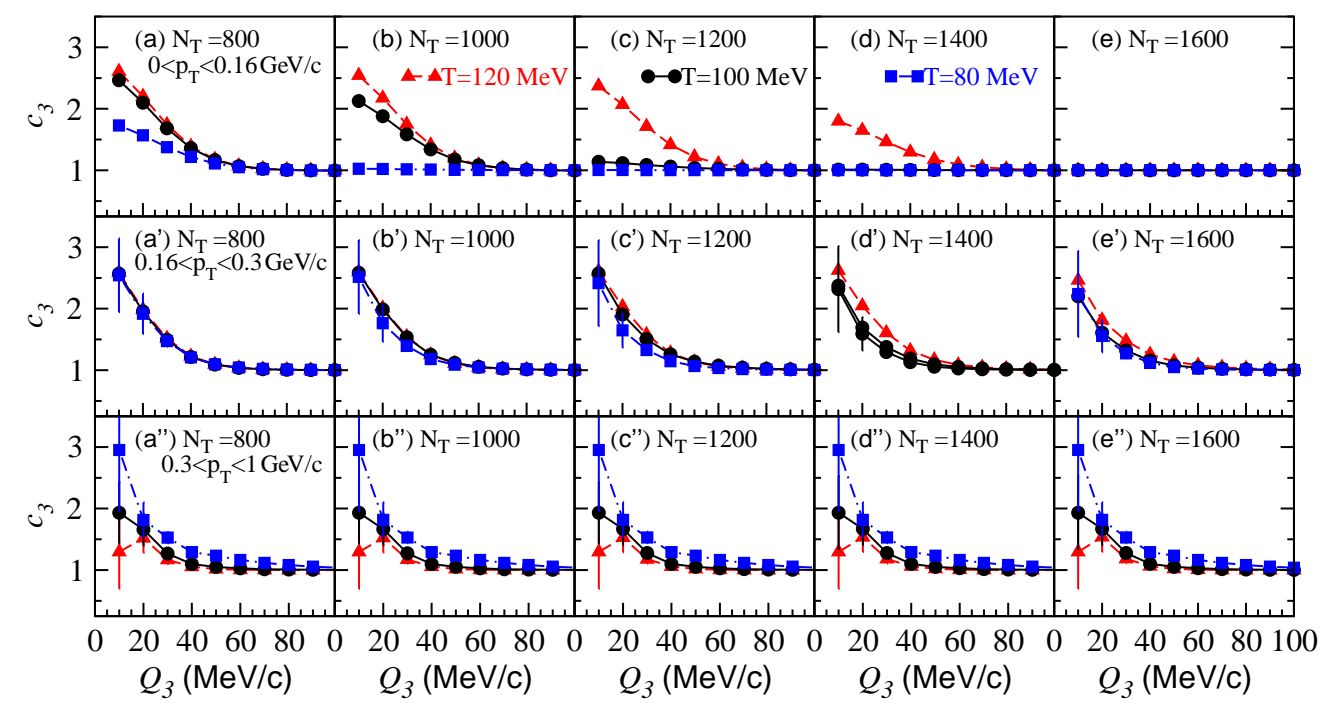

FIG. 8: Three particles cumulant correlations $c_{3}$ versus $Q_{3}$ for the large sources $K_{s}=0.40$ at various transverse momenta with different particles number $N_{T}$.

[Figs. 7(a" $\left.)-\left(e^{\prime \prime}\right)\right]$. The correlations at the intercept show that almost all particles ejected chaotically with the higher momenta of transverse even the considered sources which having the high coherence fraction due to high particles multiplicity $N_{T}$ and lower temperature $T$. It is quite obvious that the widths of the genuine correlations functions at low transverse momenta represent wider behavior than those of the correlations functions measured at highest transverse momenta especially because the pions ejected sources have wide spatial distributions for the pions ejected from the excited state. The cumulant (genuine) correlations for our considered sources at higher temperature are slightly lower than those of $T=80 \mathrm{MeV}$ due to fact that the spatial distributions are narrower at the lower temperatures for the chaos emission ejected from the excited states.

In particular, the effect of the coherence fraction on the genuine correlations appeared little as shown in the middle momentum interval $0.16<p_{T}<0.3 \mathrm{GeV} / c$ than those at lower transverse momenta because the ejection of pions from excited states are numerically larger that those at the lowest momentum. The impact of spatial distributions also play a vital role on the cumulant correlations in a particular way at various temperatures as shown in the momentum dependent middle panels.

Fig. 8 illustrate the genuine correlations functions of three particles $c_{3}$ versus relative momenta $Q_{3}$ which is invariant for the evolving sources contained pion gas with the parameter $K_{s}=0.40$ and contained the particles numbers $N_{T}=800,1200,1400,1600$. The 
cuts of the transverse momenta are imposed in our model calculations as $p_{T}<0.16 \mathrm{GeV} / c$, $0.16<p_{T}<0.3 \mathrm{GeV} / c$ and $p_{T}>0.3 \mathrm{GeV} / c$ respectively. The top horizontal panels shows the variations of the genuine cumulant correlations of three particles when all the correlations of two particles removed. The multiplicity of such bosons with different particles increased from left to right gradually to examine the characteristics of the coherence on the genuine correlations. It is obvious that at the lower transverse momenta the genuine correlations decrease significantly with increasing the numbers of particles at fixed temperature as shown in the Figs. 8(a)-(e). The genuine or pure correlations of three particles increase with rising the temperature at constant the particles numbers and decrease with lowering the temperature evidently, but the effect of coherence appears significantly at lower temperature $T=80$ $\mathrm{MeV}$ when the number of particles are 1600 due to the fact that if any two bosons ejected from the coherence state the the corresponding genuine interference of bosons appear null which cause by the Bose Einstein condensation.

One can note that the behavior of the genuine correlations is significantly different from the simple correlations of three particles because genuine correlations determine the delicate effect of coherence effectively that those of the correlations. However, the genuine correlations at the higher transverse momenta are independent on the temperature and number of particles because the particles with highest momentum come chaotically from the higher energy states which push the considered bosons to correlate quantum mechanically and appear to be the chaotic behavior and touch to the chaotically limit three as shown the last two horizontal panels of Fig. 8.

Fig. 9 shows the normalized three particles correlator $r_{3}$ versus relative momenta $Q_{3}$ for the sources with distinct temperatures $\mathrm{T}$ and particle numbers $N_{T}$. The $r_{3}$ for the given sources contained the numbers of particles $\left(N_{T}=600,800\right.$ and 1000 for the sources of small size parameter $K_{s}=0.35$ as given in the Figs. $9(\mathrm{a})-(\mathrm{c})$. The the considered correlator of the three particles decrease significantly by increasing the numbers of particles when we fixed the temperature because large numbers of particles possess the probability to reside the numbers of bosons coherently at the lower temperatures. The phenomenon of lowering the temperature at fixed number of particles $N_{T}$ cause to decrease the kinetic energy of the pions particles continuously $K . E \propto T$ and pions start to condense at the lower energy level, and produce the Bose-Einstein condensations frequently which effect the bosons normalized correlator significantly. It is more interesting to discuss that for the small sources with pa- 

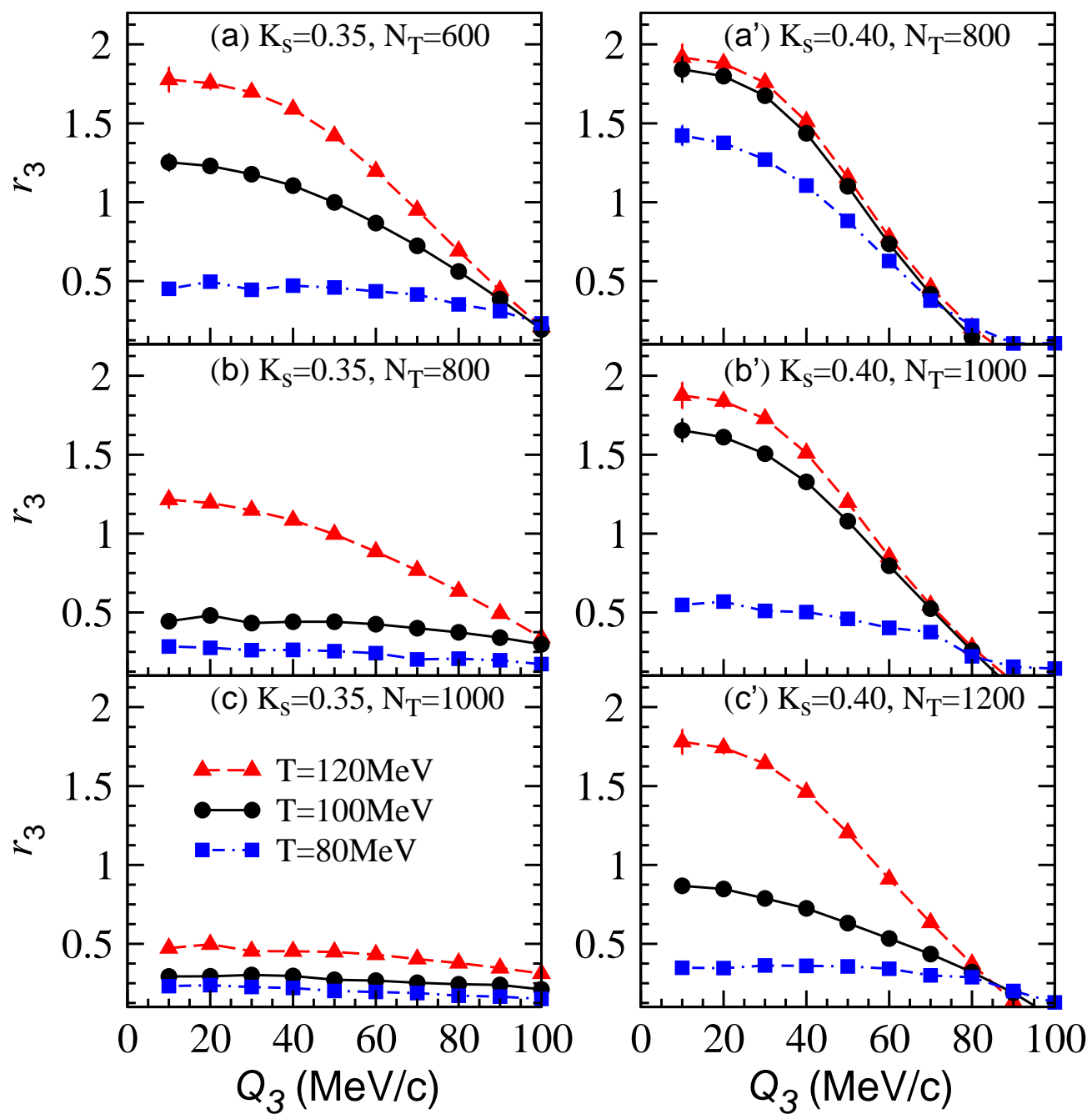

FIG. 9: Three particles correlator $r_{3}$ versus $Q_{3}$ at various temperatures with different particles number $N_{T}$.

rameter $K_{s}=0.35$ the effect of the intensity interference appear to zero when the number of particles are in the range of 1000 as shown in the Fig. 9(c). Similarly, Figs. 9( $\left.a^{\prime}\right)-\left(c^{\prime}\right)$ describe the variation of the normalized correlations of three considered bosons when the given source possess the pretty large size with the parameter $K_{s}=0.40$ ) which contains the particles numbers $N_{T}=800,1000$, and 1200 . The normalized correlators decrease by increasing $N_{T}$ because the considered source with large number of particles acquired the significant coherence effect. Such normalize correlator increase with rising the temperature for fix the number of particles $N_{T}$ because at higher temperatures the effect of coherence appears small significantly and the normalized correlator function approach to the chaotic limit two at the intercepts. 


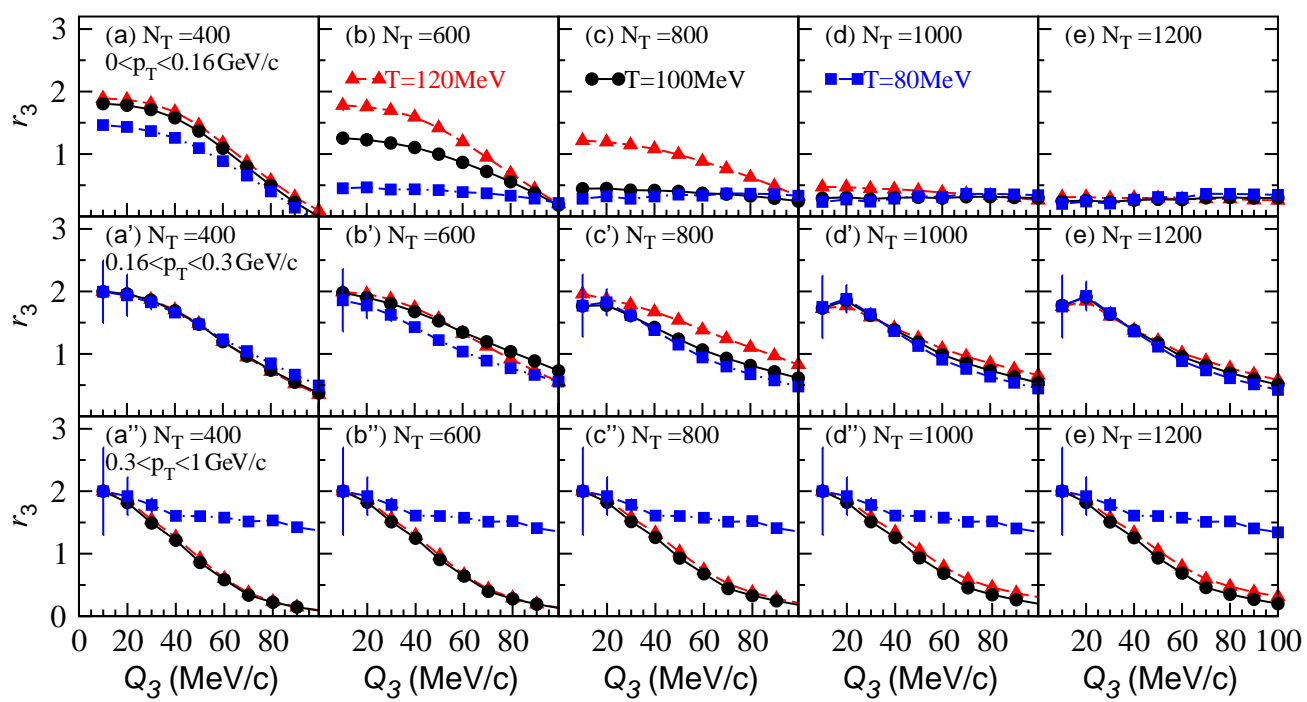

FIG. 10: Three particles correlator $r_{3}$ for the small sources $K_{s}=0.35$ versus $Q_{3}$ at various transverse momenta with different particles number $N_{T}$.

In particular, the results of $r_{3}$ are pretty suppressed at lower temperatures for the fixed number of particles $N_{T}$ for both sources. It is quite clear that the normalized corrrelators of three particles for the considered sources with $K_{s}=0.40$ have an obvious enhancement than for the small sources with the parameter considered $K_{s}=0.35$ by fix the number of particles $N_{T}$ and temperature $T$. This cause happen due to fact that the source with large parameter $K_{s}$ appear to be the large in size and their corresponding energy levels appear to be narrow which invites to the maximum boson to transport from lower energy to the higher energy states, and possessed the large characteristics lengths which tends to the lower coherence fraction. Fig. 10 presents the normalized correlator function for three particles $r_{3}$ versus relative momenta at various temperatures $\mathrm{T}$ of the particle emission sources with particles numbers $N_{T}=400,600,800,1000$ and 1200, with considered size parameter $K_{s}=0.35$. The top panels at $0<p_{T}<0.16 \mathrm{GeV} / c$ show that the correlator $r_{3}$ increase with rising the temperature and proceed to two at the high temperature regions because the emissions of particles from the chaos states. These levels of energy possess high momenta and temperatures for the system with finite and lower coherence fraction. However, such correlator decreases with increasing the quantity of particles number at the fixed transverse low momenta because the higher number of particles compelled the system towards coherence state where there is no probability of occurrence of intensity interferences. Such interesting information about coherence shown in Figs. 10(d)-(e). 


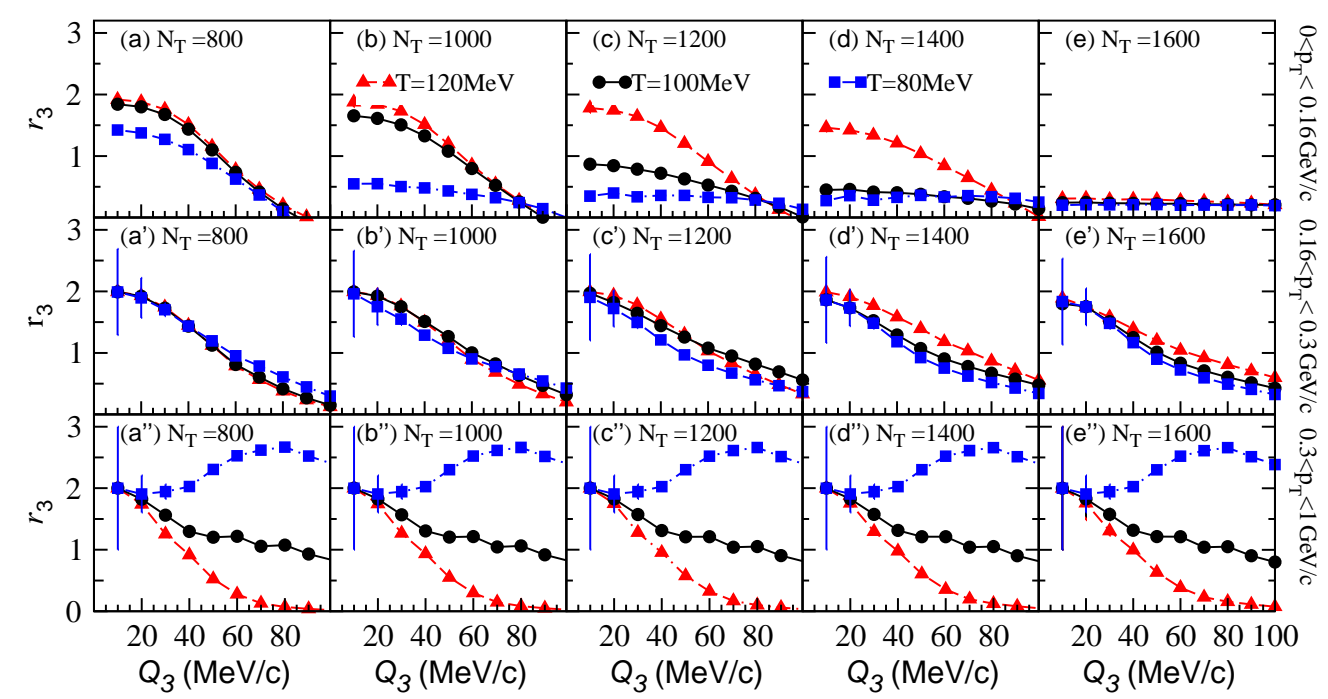

FIG. 11: Three particles correlator $r_{3}$ for large sources $K_{s}=0.40$ versus $Q_{3}$ at various transverse momenta with different particles number $N_{T}$.

The normalized correlator functions show a dramatic enhancement at high momentum regions even the temperature is pretty lower. Figs. 10 $\left(a^{\prime}\right)-\left(e^{\prime}\right)$, and Figs. 10 $\left(a^{\prime \prime}\right)-\left(e^{\prime \prime}\right)$ illustrate the correlations at highest transverse momenta regimes $0.16<p_{T}<0.3 \mathrm{GeV} / c$, and $p_{T}>0.3 \mathrm{GeV} / c$, respectively in order to examine the the effect of coherence with temperature and numbers of particles. The corresponding results express the significant chaotic limit at the intercept even in the presence of lower temperature and higher multiplicity. The values of $r_{3}$ insensitive with temperature $T$ and attain the chaotic limit two of the particle ejected source and decrease gradually at large $Q_{3}$. The reasons are quite obvious that the emission sources at higher momenta possess the lower condensation fraction. The correlators for the considered source with lower temperature $80 \mathrm{MeV}$ show an obvious pretty enhancement at large $Q_{3}$. The reason is that the spatial distribution at large momentum appeared narrow. Such behavior represents the indication of chaotic source at higher momentum regimes.

Fig. 11 shows the normalized correlator functions $r_{3}$ of three particles versus relative momenta $Q_{3}$ for the evolving sources contained pion gas with the parameter $K_{s}=0.40$ and contained the particles numbers $N_{T}=800,1200,1400$ and 1600. The cuts of the transverse momenta are used during model calculations as $p_{T}<0.16 \mathrm{GeV} / c, 0.16<p_{T}<0.3 \mathrm{GeV} / c$ and $p_{T}>0.3 \mathrm{GeV} / c$ respectively. The top horizontal panels shows the variations of $r_{3}$ with distinct temperatures, number of particles and momentum about three particles. The multiplicity of such bosons with different particles increased from left to right gradually to 
analyze the characteristics of the particle ejected sources on the normalized correlator. It is obvious that at the lower transverse momenta the normalized correlator correlations decrease significantly with increasing the numbers of particles at fixed temperature as shown in the Figs. 11(a)-(e). The results increase with rising the temperature at constant particles numbers $N_{T}$ and decrease with lowering the temperature evidently. The effect of coherence appears significantly at all temperature when the numbers of particles are 1600 due to the Bose-Einstein coherence of bosons and $r_{3}$ appear to zero.

One can note that the behavior of normalized correlators is significantly different at higher transverse momenta regimes. Middle panels illustrate the results at $0.16<p_{T}<0.3 \mathrm{GeV} / c$ and show that $r_{3}$ at the higher transverse momenta are independent on the temperature and number of particles because the particles with highest momentum come chaotically from the higher energy states which push the considered bosons to correlate quantum mechanically and appear to be the chaotic and touch to the chaotically limit two at small $Q_{3}$ regimes as shown in Figs. 11( $\left.a^{\prime}\right)-\left(e^{\prime}\right)$.

In particular, the values of $r_{3}$ are insensitive at the intercept with temperature $T$ and multiplicity $N_{T}$ in the presence of $p_{T}>0.3 \mathrm{GeV} / c$ and attain the chaotic limit two and suppress gradually at large $Q_{3}$. The reasons are quite obvious that the emission sources at higher momenta eject particles from the excited energetic states even the temperature decreases. The model results of $r_{3}$ at high transverse momenta regimes show an increase at lower temperature $80 \mathrm{MeV}$ and this enhancement increase with increasing the relative momenta $Q_{3}$ at the large regimes of $Q_{3}$ relative to the model results with the higher temperature. This factor of increasing associated to the escalation of chaos parameter with increasing the particle momentums at the wide ranging variational momentum region.

\section{SUMMARY AND CONCLUSION}

Three particles quantum statistics correlations in collisions have been investigated by difference equations and we perceive that the correlations change considerably with the variation of temperature of the particle ejecting sources. The correlations at higher temperature uniquely display the quantum interferences effect which examined with an efficient approach of pions femptoscopy for fraction order. However, we have examined that the particle production multiplicity proceed to thousands at unprecedent thermal energies and such 
extraordinary multiplicity of pions optimistically emanated the coherence radiance which decrease the correlations significantly at lower temperature and heat energy, respectively. The simple two and three particles particles interferences at the elementary level are analyzed in exploring the existence of coherence in the particle production sources at higher energies.

The normalization of three particles cumulant to the two particles comulant is quantified with $r_{3}$. We find an obvious suppression below thermal limit in the measurement of $r_{3}$ at lower temperature and momentum, respectively. Such suppression of $r_{3}$ appears to recommend coherence components to particles production in the collisions of heavy nuclei. It is crucial at lower temperature, momentum and such consideration is qualitatively compatible with the origination of Bose Einstein condensation which is contemplated to emit coherently at the lower energies.

In particular, the normalized three particles correlator $r_{3}$ is applicable for examining the thermal and coherence fractions of particle production sources created at unusual energies. We evaluated the $r_{3}$ for three pions particles at various temperatures and momentum regiom$\mathrm{s}$, and explored the consequences of the particles number and source size on the considered normalized correlator. We have observed that the normalized three particles correlator are correlated to the chaotic limit of the two particle interferences and also delicate to the thermalization of the particle emissions sources. Such correlator dwindle with dwindling the temperature $T$ but increasing at higher temperature because the source chaotic fractions increases with rising temperature $T$ and decreasing at small momentum significantly.

Specifically, the possession of $r_{3}$ on the temperature $T$ and number of particles $N_{T}$ become insensitive in the high momentum regions, which contemplate the salient features of the pion emissions sources that the high momenta particles are ejected thermally from the higher energy levels even the sources accompanied a substantial coherence fraction. Although the considered model explained the usual particle ejecting source which enlarge spherically and it produced the characteristics of the heat exchangers and coherence fractions in the collisions at the higher thermal energies. In this research work, we analyzed that the considered model confers the normalized three particle correlator in basic agreement with the practical applications of the heat exchanger with chaotic and coherence measurements in order to use in the field of thermal engineering. We have explored the full three particles correlations, cumulant correlations and three particles normalized correlator, respectively. We realized that the three particles cumulant and the normalized correlator are suppressed crucially in 
the small temperature regimes at small transverse momenta. One plausible elucidation of this consequence is that the particles emission from the radiated source which created in the collision possessed the fraction of coherence which is non-thermal.

\section{Declaration statement}

It is to make you sure that this paper is new and not submitted to any other journal. All the results included are new and not reported before.

\section{Availability of data and materials}

All data is fully available without any restriction. The data can be found inside the manuscript. No data is hidden or restricted.

\section{Competing of interests}

The authors declare that they have no known competing financial interests or personal relationships that could have appeared to influence the work reported in this paper.

\section{Funding}

Yibin University provided the financial support for this study.

\section{Authors contributions}

GB Conceptualization and writing of original draft; WA and MS project administration and writing of original draft; RA resources, writing and editing; IK literature search and data analysis. 


\section{Acknowledgments}

We are thankful to the Faculty of Science, Yibin University, Yibin, China for support provided for this research.

[1] M. Gyulassy, S. K. Kauffmann and L. W. Wilson, Phys. Rev. C 20, 2267 (1979).

[2] U. A. Wienemann and U. Heinz, Phys. Rep. 319, 145 (1999).

[3] R. M. Weiner, Phys. Rep. 327, 249 (2000).

[4] T. Csorgo, Acta Phys. Hung. A 15 (2002).

[5] M. A. Lisa, S. Pratt, R. Soltz, and U. Wiedemann, Annu. Rev. Nucl. Part. Sci. 55, 357 (2005).

[6] M. M. Aggarwal et al. [WA98 Collaboration], Phys. Rev. C 67, 014906 (2003).

[7] S. Pratt, Phys. Lett. B 301, 159 (1993).

[8] T. Csörgő and J. Zimányi, Phys. Rev. Lett. 80, 916 (1998).

[9] D. Gangadharan, Phys. Rev. C 92 (2015).

[10] G. Goldhaber, S. Goldhaber, W. Y. Lee and A. Pais, Phys. Rev. 120 (1960).

[11] I. V. Andreev, M. Plumer and R. M. Weiner, Int. J. Mod. Phys. A 8 (1993).

[12] H. Boggild et al. [NA44 Collaboration], Phys. Lett. B 455, 77 (1999).

[13] J. Adams et al. [STAR Collaboration], Phys. Rev. Lett. 91, 262301 (2003).

[14] R. Willson [STAR Collaboration], Nucl. Phys. A 715, 619 (2003).

[15] B. B. Abelev et al. [ALICE Collaboration], Phys. Rev. C 89 (2014).

[16] U. W. Heinz and Q. H. Zhang, Phys. Rev. C 56 (1997).

[17] U. Ornik, M. Plumer and D. Strottmann, Phys. Lett. B 314 (1993).

[18] J. P. Blaizot, F. Gelis, J. Liao et al, Nucl. Phys. A 904-905 (2013).

[19] E. Ikonen, Phys. Rev. C 78 (2008).

[20] C. Y. Wong and W. N. Zhang, Phys. Rev. C 76, 034905 (2007).

[21] J. Liu, P. Ru, W. N. Zhang, C. Y. Wong, J. Phys. G 41, 125101 (2014).

[22] M. Naraschewski and R. J. Glauber, Phys. Rev. A 59, 4595 (1999).

[23] C. Y. Wong, W. N. Zhang, J. Liu, and P. Ru, arXiv:1501.04530.

[24] Y. M. Liu, D. Beavis, S. Y. Chu et al, Phys. Rev. C 34, 1667 (1986).

[25] U. Heinz and Q. H. Zhang, Phys. Rev. C 56, 426 (1997); 
U. Heinz and A. Sugarbaker, Phys. Rev. C 70, 054908 (2004).

[26] Ghulam Bary, Wei-Ning Zhang, Peng Ru, Jing Yang, Chinese Phys. C 45, 024106 (2021).

[27] Ghulam Bary, Muhmmad Imran, Arslan Muhammad et al, Journal of Saudi Chemical Society, 101260 (2021).

[28] Sajid Muhammad, Zhao Xuebing and Liu Dehua, Green chemistry 20, 5427-5453 (2018).

[29] Sajid Muhammad, Bai Yuchen, Liu Dehua et al, Arabian Journal of Chemistry 13 (2020).

[30] H. Bøggild et al. (NA44 Collaboration), Phys. Lett. B 455, 77 (1999);

I. G. Bearden et al. (NA44 Collaboration), Phys. Lett. B 517, 25 (2001).

[31] M. M. Aggarwa et al. (WA98 Collaboration), Phys. Rev. Lett. 85, 2895 (2000);

M. M. Aggarwa et al. (WA98 Collaboration), Phys. Rev. C 67, 014906 (2003).

[32] J. Adams et al. (STAR Collaboration), Phys. Rev. Lett. 91, 262301 (2003).

[33] B. Abelev et al. (ALICE Collaboration), Phys. Rev. C 89, 024911 (2014).

[34] R. Ahmad, A. Farooqi, J. Zhang et al App. Mathematics and Nonlinear Sciences 4, 249-260 (2019).

[35] R. Ahmad, A. Farooqi, J. Zhang et al, IEEE access 8, 141057-141065 (2020).

[36] A. Farooqi, R. Ahmad, H. Alotaibi et al, Results in Physics 21, 103756 (2021).

[37] Ahmad Hijaz, Rafiq Muhammad, Cesarano Clemente et al, Earthline Journal of Mathematical Sciences 3, 229-247 (2020).

[38] Khan Zareen A, Ahmad Hijaz, Fractals 7, (2021).

[39] Abouelregal Ahmed E, Ahmad Hijaz, Nofal Taher A et al, Modern Physics Letters, 2150297 (2021).

[40] Ru Peng, Bary Ghulam and Zhang Wei-Ning, Physics Letters B 777, 79-85 (2018).

[41] Karaca Yeliz, Zhang Yu-Dong, Muhammad Khan, Expert Systems with Applications 144, $113098(2020)$. 